\title{
BlackBox TestBox: uma ferramenta baseada em virtualização para testes de caixa-preta
}

\author{
Carlos A M de S Teles ${ }^{1}$, Carlos Roberto Viana ${ }^{1}$, Raphael Carlos Santos Machado ${ }^{1,2}$ \\ ${ }^{1}$ CEFET-RJ - PPCIC \\ ${ }^{2}$ Inmetro - Instituto de Metrologia, Qualidade e Tecnologia \\ \{carlos.teles, carlos.filho\}@eic.cefet-rj.br, rmachado@inmetro.gov.br
}

\begin{abstract}
TIC assets are hardware and software capable of performing computational processing and are involved in information technology and data communication activities. To verify compliance with these requirements, through systematic testing, there are tests called Conformity Assessment Programs (PACs). Our proposal is the development of a virtualization-based TIC asset testing tool that enables the creation of test environments, including physical and virtual devices, which takes into account the Black Box tests, aim to identify hardware and software failures. Software using Information Security and Performance monitors.
\end{abstract}

Resumo. Ativos de TIC são o hardware e software capazes de executar processamento computacional e que estejam envolvidos em atividades de tecnologia da informação e comunicação de dados. Para verificar o atendimento a requisitos destes, por meio de testes sistemáticos, há os ensaios chamados de Programas de Avaliação da Conformidade (PACs). Nossa proposta é pelo desenvolvimento de uma ferramenta para testes de ativos de TIC baseada em virtualização, que possibilita a criação de ambientes de teste, inclusive utilizando dispositivos físicos e virtuais, que, leva em consideração os ensaios Black Box, visam identificar falhas em Hardware e Software utilizando-se de monitorações de Segurança da Informação e Desempenho.

\section{Introdução}

A segurança dos atuais ambientes tecnológicos é altamente dependente do adequado comportamento dos diversos ativos de tecnologia da informação e de comunicações que os compõem - onde ativos de tecnologia da informação e de comunicações (ativos de TIC) são utilizados para contemplar todo o tipo de hardware e software capaz de executar processamento computacional e que esteja envolvido em atividades de tecnologia da informação e de comunicações. O termo TIC refere-se à conjugação da tecnologia computacional com a tecnologia das telecomunicações e tem nas redes de computador, e principalmente na Internet, a sua maior expressão [Miranda 2007]. De fato, o funcionamento não adequado de um elemento de um sistema computacional, sendo hardware ou software, pode comprometer toda a segurança. É fundamental, portanto, que se desenvolvam mecanismos que permitam averiguar e atestar que ativos de TIC atendem a requisitos desejáveis de segurança. Tal objetivo, no entanto, é um enorme desafio, na medida em que os ativos de TIC usados nos atuais sistemas 
computacionais tornam-se cada vez mais complexos e estão inseridos em atividades cada vez mais críticas e relevantes para o bem-estar da sociedade.

Um dos grandes desafios na avaliação de segurança de ativos de TIC é a verificação de atendimento de requisitos quando não se dispõe de todos os documentos de engenharia, tais como diagramas elétricos, documentos de engenharia de software e código fonte. Neste caso, é necessário basear a avaliação da conformidade na realização de ensaios do tipo denominado caixa preta, ou seja, testes puramente "comportamentais". É preciso garantir a conformidade do comportamento do ativo mesmo não tendo acesso aos detalhes de sua especificação. A pergunta-chave é: como garantir que, em condições específicas - e não conhecidas pelo avaliador - o ativo de TIC não poderá exibir comportamento indesejado? A grande dificuldade é o fato de que, tipicamente, eventuais funcionalidades maliciosas inseridas no ativo sob avaliação só irão ser acionadas por meio da reprodução de condições muito especiais - naturalmente, conhecidas apenas pelo usuário mal-intencionado que inseriu tal funcionalidade maliciosa. De fato, a história recente do mundo da avaliação da conformidade está repleta de exemplos, nos quais ativos de TIC eram capazes de "reconhecer" que estavam sob avaliação e, neste momento, exibir o comportamento com funcionalidades maliciosas. O exemplo mais emblemático, neste sentido, talvez seja o dos carros da Volkswagen com motor a diesel TDI. A empresa declarou ao mundo em 18 de setembro de 2015 [Blackwelder, Coleman, Colunga-Santoyo, Harrison e Wozniak 2016] com sua resposta ao aviso da Agência de Proteção Ambiental dos EUA (EPA) de que os veículos "limpos a diesel" da empresa estavam violando a Lei do Ar Limpo dos EUA. Em sua resposta, a montadora alemã admitiu ter equipado sua linha de motores a diesel com injeção direta turbinada (TDI) com um dispositivo que se destinava a "ignorar" os elementos inoperantes do sistema de controle de emissão de um veículo durante o teste de emissões. Os relatórios iniciais sugeriram que aproximadamente 500.000 carros a diesel da Volkswagen, modelo de 2009 a 2011, foram afetados pelo dispositivo. Este caso possuiu desdobramentos, pois, o sistema foi vendido a outras montadoras e o número estimado de veículos afetados cresceu para 11 milhões de veículos, a partir de um cruzamento de várias marcas em todo o mundo. Se empresas como a Volkswagen são capazes de desenvolver mecanismos para burlar mecanismos de avaliação da conformidade por meio de manipulação de software, podemos concluir que é fundamental desenvolver metodologias que permitam avaliar ativos de TIC mesmo sem acesso aos seus detalhes de concepção e projeto.

As contribuições do presente trabalho são enumeradas a seguir: (i) Desenvolvimento de um ambiente de testes de ativos de TIC baseado em ferramentas de virtualização - denominamos a ferramenta BlackBox TestBox. O BlackBox TestBox se diferencia das propostas observadas na literatura, na medida em que ele possibilita a instanciação de sofisticados ambientes de teste. $O$ ambiente está disponibilizado publicamente na forma de um produto de software em https://github.com/carlosteles/etsg/, para que os experimentos possam ser reproduzidos pela comunidade e; (ii) Construção de uma base de dados de referência para o estudo de ataques a ativos de TIC a partir do tráfego capturado nos experimentos.

A ferramenta BlackBox TestBox deve ser utilizada para testes de ativos de TIC baseada em virtualização. Este se diferencia das propostas observadas na literatura, na medida em que ele possibilita a instanciação de sofisticados ambientes de teste, 
inclusive com o uso de dispositivos virtualizados. O ambiente foi disponibilizado publicamente para que os experimentos possam ser reproduzidos pela comunidade acadêmica. Adicionalmente, a disponibilidade de uma ferramenta de teste com as características do BlackBox TestBox viabiliza o desenvolvimento de uma metodologia para a realização de ensaios sistemáticos com foco em segurança. Nossa proposta se diferencia das observadas na literatura, uma vez que os testes de segurança permitem avaliar tanto as falhas de segurança que permitem que os ativos sofram ataques quanto permitem identificar eventuais comportamentos maliciosos por parte do próprio dispositivo em avaliação.

\section{Conceitos Fundamentais}

\subsection{Segurança da Informação}

Conforme identificado na Introdução, a Segurança da Informação tornou-se relevante na maioria das organizações. Seu objetivo é assegurar a proteção dos sistemas de informação e aos dados, de acesso não autorizado, uso, divulgação, interrupção, modificação ou destruição, além de diminuir os danos causados ao prevenir e minimizar o impacto de incidentes de segurança sobre os ativos de TIC. [Nieles e Pillitteri 2017]. Esta possui três propriedades principais: (i) Confidencialidade, para limitar o acesso à informação somente aos agentes autorizados; (ii) Integridade, para preservar que as informações tenham suas características fiéis à sua origem e que qualquer alteração durante o processo seja realizada com autorização e controle e; (iii) Disponibilidade, para garantir que a informação esteja acessível aos autorizados sempre que precise ser resgatada. [Von Solms 1998]

\subsection{Virtualização}

A virtualização foi desenvolvida primeiramente pela IBM Corporation em 1960, originalmente para dividir o mainframe em várias instâncias lógicas e para rodar em um único hardware físico como o host. Esse recurso foi inventado porque a manutenção dos computadores mainframe maiores tornou-se incômoda e alto gasto. Assim, a máquina virtual foi criada no mainframe e apenas no inicio dos anos 2000, foi desenvolvido para a plataforma x86. Avanços tecnológicos em hardware e software tornam as máquinas virtuais estáveis, acessíveis e oferecem um enorme valor, dada a implementação correta. Assim, a virtualização tornou-se parte integrante da maioria das organizações e está cada vez mais difundida em vários setores. Isso reduziu os custos de TIC e aumentou consideravelmente sua receita. [Agesen, Garthwaite, Sheldon e Subrahmanyam 2012] [Francia III, Garrett e Brookshire 2012] [Ameen e Hamo 2013]

\subsection{Teste Black Box e White Box}

$\mathrm{Na}$ área de software, o principal objetivo dos testes é identificar falhas para que os mesmos possam ser corrigidos. O teste de software também é utilizado para testá-lo quanto a outros fatores de qualidade de software como confiabilidade, usabilidade, integridade, segurança, capacidade, eficiência, portabilidade, manutenção, compatibilidade, etc. As duas técnicas mais importantes usadas para encontrar erros são os testes de Black Box e os White Box. [Kumar, Singh e Dwivedi 2015] 
A técnica para testes White Box é uma atividade investigativa detalhada da lógica interna e estrutura do software, via código-fonte, e hardware, via esquemas e diagramas elétricos Para tal é preciso que o testador tenha todo o conhecimento do ativo de TIC a ser testado. Para testes Black Box é uma atividade técnica de teste sem ter qualquer conhecimento, via código-fonte, ou acesso ao funcionamento da lógica interna e estrutura do software. Esta técnica examina os aspectos fundamentais de um sistema, via software e hardware, de entradas e saídas aguardadas do ativo de TIC a ser testado. Uma das características, segundo [Sjöberg, Zhang, Ljung, Benveniste, Delyon, Glorennec e Juditsky 1995], é que nenhum resultado deve ser excluído dos testes, pois, há um espectro muito rico das descrições de modelos possíveis que devem ser tratados.

\section{Trabalhos Relacionados}

Nesta seção serão apresentados trabalhos relacionados a esta pesquisa. Dado o fato que a mesma é uma combinação de conceitos de: Segurança da Informação, Virtualização e Teste BlackBox, relacionamos os trabalhos destas três áreas.

Para avaliar os diferentes trabalhos relacionados à nossa pesquisa foi feita uma busca sistemática, data de 09 de Novembro de 2018 na base de dados do Scopus a partir das palavras-chave citadas acima. Obtivemos o retorno de sete artigos e após a leitura do resumo destes, identificamos e classificamos os seguintes trabalhos.

Com as palavras-chave "BlackBox" e "Virtualização", o trabalho de [Taheri, Zomaya, e Kassler 2017], indica que nenhum agente é necessário a ser instalado dentro de máquinas virtuais para monitoramento; as medidas são feitas no hypervisor, o que indica o uso de virtualização completa; fornece uma abordagem sistemática para calcular a sensibilidade das máquinas virtuais aos seus recursos (CPU, Memória e Disco). Medição das máquinas virtuais é um processo classificado como Black-Box.

Com as palavras-chave "Black-Box", "Segurança da Informação" e "monitoramento", o trabalho de [Bauer, Heseding e Flittner 2017], leva em consideração que o orçamento é muito limitado, assim, seus custos operacionais devem ser bem controlados. O EarlyDrop é um mecanismo de defesa DDoS baseado no monitoramento transparente, assumindo que os sistemas de mitigação como Black-Box.

Com as palavras-chave "Segurança da Informação" e "monitoramento", o trabalho de [Ibidunmoye, Lakew e Elmroth 2017] usa o Teste de Black-Box para detecção de anomalias em rede, ou melhor, anomalias em ativos de TIC. Estas são detectadas pela descoberta de alterações no padrão de consumo de recursos (CPU, Memória, Tráfego de rede) temporais dos nós de um datacenter.

Em paralelo a busca sistemática acima, fizemos uma pesquisa ad hoc pvisando encontrar às iniciativas relacionados a Segurança da Informação, Virtualização e Teste BlackBox, que poderiam estar relacionadas e encontramos outros trabalhos.

O Docker Security Playground segundo [Perrone e Romano 2017] possui uma arquitetura que utiliza uma abordagem baseada em microsserviços para construir infraestruturas de rede complexas especificamente adaptadas ao estudo da segurança de rede com uma abordagem prática. De todos os trabalhos relacionados, é o que possui características, em relação ao ambiente de virtualização, mais próximas, a nossa pesquisa. Apontamos como dois que não se aproximam como: (i) utiliza somente 
arquitetura x86 de 32 bits, não instanciando outras (arm, mips, x86_64 e etc.) e (ii) não possui um ambiente de monitoração associado.

\section{Proposta}

Nossa proposta se caracteriza pelo desenvolvimento de uma ferramenta para testes de ativos de TIC baseada em virtualização, que possibilita a instanciação de ambientes de teste, inclusive utilizando dispositivos físicos e virtuais, que, leva em consideração os ensaios Black Box, visam identificar falhas em Hardware e Software utilizando-se de monitorações de Segurança da Informação e Desempenho. A ferramenta possui dois módulos: Ambiente de Monitoração e Ambiente de Virtualização.

Um dos itens mandatórios é que os Ambientes devem estar no mesmo segmento de rede para um bom desempenho de monitoração. O Ambiente de Monitoração ficará coletando os dados dos ativos de TIC enquanto conectados a rede, de forma a detectar não conformidades, através da identificação da mudança de estado do tráfego de rede, juntamente com alertas e eventos gerados através de um mecanismo de detecção de ameaças de rede, através dos módulos IDS, IPS e NSM. E o Ambiente de Virtualização poderá possuir ativos de TIC físicos e virtuais, utilizados para simular um ambiente real e operacional. Os ativos de TIC que serão testados também estarão conectados a este ambiente, de forma que todos estejam monitorados. Selecionamos a virtualização completa hospedada. Os Ambientes de Monitoração e Virtualização foram pensados para ser leves, podendo ser carregados a partir de um pendrive.

\section{Implementação}

Abordaremos agora a implementação dos ambientes. Primeiramente apresentando os componentes e posteriormente como atuarão entre si.

\subsection{Ambiente de Monitoração de Segurança e Desempenho}

Este ambiente pode ser dividido em duas partes com: Ambiente de Monitoração de Segurança, com Suricata e Evebox e; Ambiente de Monitoração de Desempenho, com Telegraf, Influxdb e Grafana (TIG).

Em nosso trabalho utilizaremos o Suricata, pois, o mesmo acumula as funções de IDS, IPS e NSM. É um sistema que pode ser usado para inspecionar o tráfego da rede usando uma linguagem de regras e assinaturas. Utilizado para detecção de invasão de rede, prevenção de invasão de rede e prevenção de monitoramento de segurança. Este é capaz de lidar com tráfego alto, exibi-lo na tela e também enviar alertas por e-mail; suporta Multi-Threading, para que você possa usar mais de uma CPU por vez; fornece aceleração de hardware incorporada, utilizando-se de placas gráficas para inspecionar tráfego da rede; suporte a diversos Sistemas Operacionais. [Suricata 2018]

EveBox é uma ferramenta de gerenciamento de eventos e alertas baseada na web para eventos gerados pelo mecanismo de detecção de ameaças da rede Suricata. É possível configurar a exportação de dados para buscas simples com o banco de dados SQLite ou PostgreSQL. Utilizaremos esta abordagem, pois, não exige conhecimento de configuração, além de transformar a visualização de dados leve, assim como a possibilidade de exportação dos mesmos. [Evebox 2018] 
O Telegraf e o Influxdb são componentes do ecossistema TICK, desenvolvidos pela empresa InfluxDATA, e em nossa pesquisa os utilizaremos na versão código aberto. O Telegraf é um agente baseado em servidores para coletar métricas e dados. Possui integrações para obter uma variedade de métricas, eventos e registros diretamente dos contêineres e sistemas ao qual está em execução.O InfluxDB é um banco de dados de séries temporais de código aberto e sem esquema. Ele está escrito na linguagem de programação Go. Possui uma linguagem de consulta semelhante a SQL. [Naqvi, Yfantidou, e Zimányi 2017]

O componente Grafana, que mostrará os dados coletados via Telegraf e Influxdb, é uma plataforma aberta para análises e monitoramento de dados. Com o Grafana é possível consultar, visualizar, alertar e entender suas métricas, independentemente de onde elas estejam armazenadas. [Naqvi, Yfantidou, e Zimányi 2017]

\subsection{Ambiente de Virtualização para ensaios}

Existem diversas opções para Virtualização de Ambientes, dentre os conceitos apresentados, a virtualização completa hospedada é a que se adéqua a nossa pesquisa e os softwares Oracle VirtualBox e o QEMU as possuem.

O Oracle VirtualBox é um software de virtualização da Oracle Corporation. Este visa criar ambientes virtualizados para instalação de sistemas operacionais distintos. Possui um desenho modular com interfaces de programação internas bem definidas e um desenho cliente/servidor. Uma de suas características, limitantes para a pesquisa, é o suporte apenas a arquitetura x86 e x86-64. [VirtualBox 2018]

O QEMU é um software emulador e virtualizador de máquinas, é executado em vários sistemas operacionais hospedados, como Linux, Windows e Mac OS X, podendo as CPUs hospedadas e as convidadas ser diferentes. Seu principal uso é hospedar um sistema operacional convidado, como o Windows no Linux ou o Linux no Windows. É utilizado principalmente para testar o resultado de compiladores cruzados ou para testar o emulador de CPU sem precisar iniciar uma máquina virtual completa. Algumas das características: Emulador de CPU e dispositivos; Debugger; Interface gráfica e; Multi arquitetura (x86, x86-64, mips, arm, entre outros). [Bellard 2005]

\section{Validação}

Para validar o funcionamento dos ambientes BlackBox TestBox, demonstramos o seu funcionamento em três cenários: (i) Internet; (ii) Interno e; (iii) Escritório em casa. Cada um caracteriza-se por uma topologia distinta e um conjunto específico de dispositivos.

\subsection{Cenários}

No cenário Internet os ativos de TIC são: um Switch, uma câmera IP e dois servidores. Como o nome já diz este ambiente está conectado diretamente na Internet. Há um servidor com o Ambiente de Monitoração instalado e possui duas placas de rede. Uma provê conectividade ao ambiente e a outra placa de rede recebe todo o tráfego de rede para análise. Isto é feito via Port Mirroring no Switch para o servidor do Ambiente de Monitoração. Há outro servidor com a função de Honeypot. Este cenário não possui nenhum firewall ou dispositivo protegendo os componentes. Isto ocorre exatamente para que se receba alto tráfego de rede e tentativas de incidentes de segurança. 
Para o cenário Interno os ativos de TIC são: um Roteador Wifi, um Smartphone, um servidor e um desktop e mais máquinas virtuais. É uma rede local. Um dos servidores está com o de Ambiente de Monitoração instalado e possui três placas de rede. Além disso, foram instanciados via QEMU cinco máquinas virtuais na mesma rede. Neste servidor uma placa de rede provê conectividade ao ambiente e a outra placa de rede recebe todo o tráfego de rede para análise pelo Ambiente de Monitoração. Isto é feito via Ettercap no servidor. Um Smartphone conectado a rede via Wifi. O desktop também possui duas máquinas virtuais instanciadas via Oracle VirtualBox, na mesma rede e atua na rede também como cliente. Este cenário que possui firewall protegendo os componentes.

Para o cenário Escritório em casa os ativos de TIC são: um Roteador Modem Wifi, três Smartphones, um servidor, um desktop, uma Impressora Multifuncional, um extensor de rede, uma SmartTV e um decodificado de Tv a Cabo. Este ambiente é uma rede local. O servidor possui o Ambiente de Monitoração de Segurança e Desempenho e uma placa de rede. Neste servidor uma placa de rede provê conectividade ao ambiente e recebe todo o tráfego de rede para análise pelo Ambiente de Monitoração, que é feito via Ettercap. Uma das características deste cenário é que possui firewall protegendo os componentes no Roteador Modem. Ainda assim, recebemos algum tráfego de rede da Internet controlado. Isto ocorre para que seja que seja possível avaliar o tráfego de rede local e possíveis tentativas de incidentes de segurança.

\subsection{Execução dos Testes}

O cenário Internet, a coleta dos dados iniciou-se em 27 de Junho de 2018 e fizemos uma data para corte em 30 de Setembro de 2018. Os dados foram coletados utilizando-se espelhamento de porta do Switch para uma porta em separado do servidor de monitoração. Assim, foi possível analisar o tráfego de toda a rede pelos componentes. Este é um ambiente que não possui nenhum tipo de segurança da informação, está conectado diretamente a Internet.

Para o cenário Interno, a coleta dos dados iniciou-se em 27 de Junho de 2018 e fizemos uma data para corte em 30 de Setembro de 2018. Os dados foram coletados utilizando-se o software Ettercap, onde o tráfego passou pelo desktop de monitoração. Assim, foi possível analisar o tráfego de toda a rede pelos componentes. Este é um ambiente que possui diversos níveis de firewall, ou seja, tem segurança da informação reforçada. Esta conectado a Internet e possui um NAT via IPv4.

Para o cenário Escritório em Casa, a coleta dos dados iniciou-se em 01 de Setembro de 2018 e fizemos uma data para corte em 30 de Setembro de 2018. Os dados foram coletados utilizando-se o software Ettercap, onde o tráfego passou pelo desktop de monitoração. Assim, foi possível analisar o tráfego de toda a rede pelos componentes. Este é um ambiente que possui pelo menos um firewal, ou seja, tem segurança da informação. Está conectado a Internet e possui um NAT via IPv4.

\subsection{Resultados}

Os resultados obtidos por meio do monitoramento de dispositivos nos três cenários, nos permitiu demonstrar a viabilidade do BlackBox TestBox à realização de ensaios de segurança baseados em testes de caixa-preta. Ainda que não tenhamos identificado 
falhas e vulnerabilidades "novas", identificamos comportamentos "suspeitos" por parte de ativos de TIC. Assim, até o momento, com os dados analisados, fomos bemsucedidos em identificar não conformidades em todos os cenários, através do Ambiente de Monitoração de Segurança e Desempenho. Abaixo, uma breve análise por cenário.

O cenário Interno apresentou (Figura 1a) a mensagem "TLS invalid handshake message". Esta mensagem é um alerta de falso positivo, ou ainda, em função de estar executando o Ettercap para capturar o tráfego. Também apresentou (Figura 1b) eventos do tipo FILEINFO e FLOW, com troca de informações entre o servidor hospedeiro do Oracle VirtualBox e o Roteador Modem Wifi.

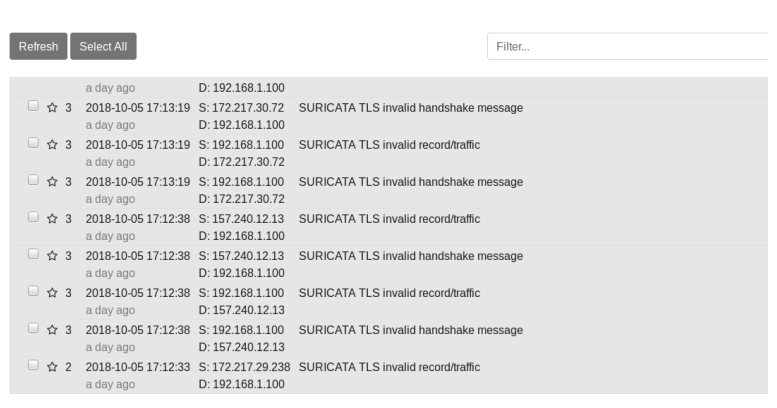

Figura 1a - Evebox 'TLS'

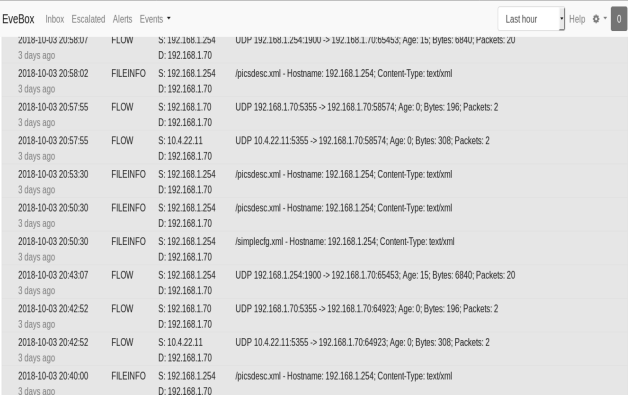

Figura 1b - Evebox 'FLOW'

Verificamos o ambiente de coletas de desempenho para realizar correlações sobre os eventos Suricata/Evebox com TIG. Nestas consultas, expomos 30 dias, pois o ambiente se apresentou bastante estável. Os dados de CPU, Processos e Load Averege, Memória e dados de tráfego de rede mantiveram-se sem variações.

Com o cenário Escritório em Casa identificamos acessos indevidos de uma aplicação, que apesar de instalada, não era mais utilizada há mais de um ano. Identificamos a aplicação através de um evento do tipo DNS. É a chamada ' www.gastecnologia.com.br'(Figura 2a). E continuando as consultas, identificamos mais acessos indevidos da aplicação, através de mais um evento do tipo DNS. Esta é a chamada 'guardiao.itau.com.br' (Figura 2b).

A partir, principalmente do primeiro evento 'guardiao.itau.com.br', fomos verificar o ambiente de coletas de desempenho para realizar correlações sobre os eventos Suricata/Evebox com TIG. As informações capturadas e nestas consultas, de CPU, Processos e Load Averege, Memória e dados de tráfego de rede não há variação. Constata-se apenas que houve tráfego de rede.

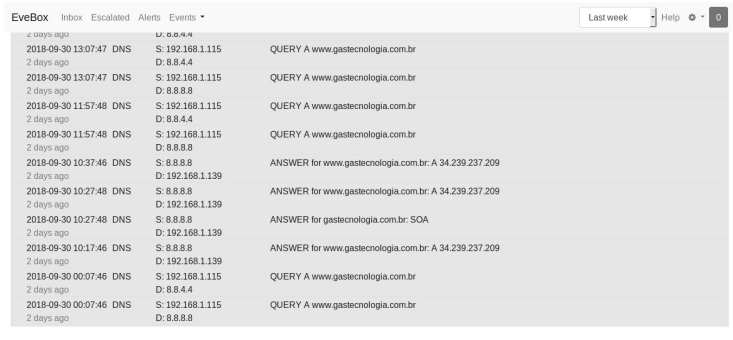

Figura 2a - Evebox 'gastecnologia'

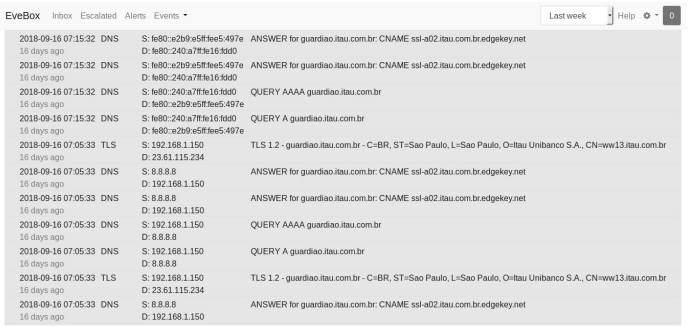

Figura 2b - Evebox 'guardiao' 
Abaixo os resultados do cenário Internet. Neste ambiente, em uma das consultas ao Suricata/Evebox, verificamos tentativas de uso do código malicioso do evento de segurança conhecido como Mirai, ao Honeypot. Além de tentativa de TELNET ao mesmo (Figura 3a). E em mais uma consulta, agora na Câmera IP, utilizando-se o IPv4 da mesma como origem do tráfego de rede (Figura 3b), há eventos do tipo DNS, identificando consultas pela Câmera IP a um servidor pelos nomes 'p2p10.cloudlinks.cn', 'p2p9.cloudlinks.cn', e etc.

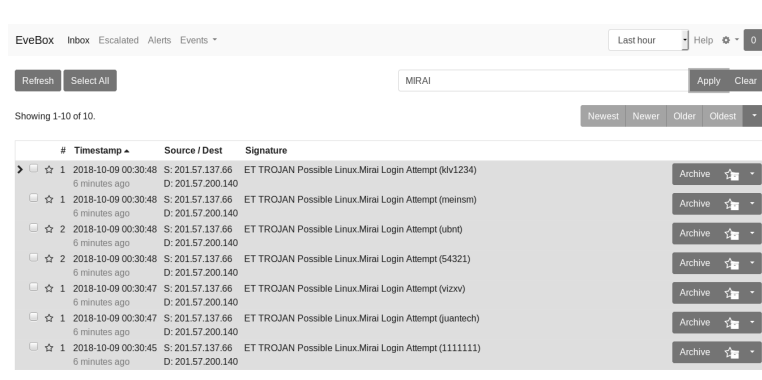

Figura 3a - Evebox 'Mirai'

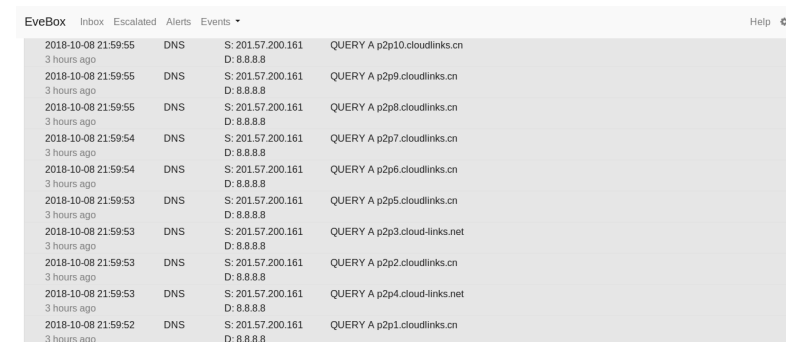

Figura 3b - Evebox 'Câmera IP'

\section{Conclusão}

Os resultados obtidos por meio do monitoramento dos ativos de TIC nos três cenários, nos permitiu demonstrar a viabilidade do BlackBox TestBox à realização de ensaios de segurança baseados em testes de caixa-preta. Ainda que não tenhamos identificado falhas e vulnerabilidades "novas", identificamos comportamentos "suspeitos" por parte de ativos de TIC.

\section{Trabalhos Futuros}

Com os resultados obtidos por meio da ferramenta BlackBox TestBox acredita-se que sejam trabalhos futuros pertinentes: (i) automatizar a extração e carga dos dados coletados, (ii) criar uma interface para consulta unificada dos dados extraídos, (iii) utilizar aprendizagem de máquina para melhorar analise dos dados, (iv) acrescentar um novo módulo (https://molo.ch) para integrar a análise de pacotes (v) carregar o ambiente de virtualização em pendrive.

\section{Agradecimento}

Este trabalho é parcialmente apoiado pelo projeto SHCDCiber.

\section{Referências}

Agesen, O., Garthwaite, A., Sheldon, J., \& Subrahmanyam, P. (2010). The evolution of an x86 virtual machine monitor. ACM SIGOPS Operating Systems Review, 44(4), 3 18.

Ameen, R. Y., \& Hamo, A. Y. (2013). Survey of server virtualization. arXiv preprint arXiv:1304.3557.

Bauer, R., Heseding, H., \& Flittner, M. (2017, October). EarlyDrop: A Trade-off Driven DDoS Defense Mechanism for Software-Defined Infrastructures. In Local Computer Networks (LCN), 2017 IEEE 42nd Conference on (pp. 207-210). IEEE. 
Bellard, F. (2005, April). QEMU, a fast and portable dynamic translator. In USENIX Annual Technical Conference, FREENIX Track (Vol. 41, p. 46).

Blackwelder, B., Coleman, K., Colunga-Santoyo, S., Harrison, J. S., \& Wozniak, D. (2016). The Volkswagen Scandal.

Francia III, G. A., Garrett, A., \& Brookshire, T. (2012). Virtualization for a CyberSecurity Laboratory. In Proceedings of the International Conference on Frontiers in Education: Computer Science and Computer Engineering (FECS) (p. 1). The Steering Committee of The World Congress in Computer Science, Computer Engineering and Applied Computing (WorldComp).

Evebox (2018). The Inbox for your Suricata Events. Disponível em: https://www.evebox.org/. - Ago 2018.

Ibidunmoye, O., Lakew, E. B., \& Elmroth, E. (2017). A Black-box Approach for Detecting Systems Anomalies in Virtualized Environments. In 2017 IEEE International Conference on Cloud and Autonomic Computing (ICCAC 2017), Tucson, Arizona, USA, 18-22 September 2017 (pp. 22-33). IEEE.

Kumar, M., Singh, S. K., \& Dwivedi, R. K. (2015). A Comparative Study of Black Box Testing and White Box Testing Techniques. International Journal of Advance Research in Computer Science and Management Studies, 3(10).

Miranda, G. L. (2007). Limites e possibilidades das TIC na educação. Sísifo. Revista de Ciências da Educação, 3, 41-50.

Naqvi, S. N. Z., Yfantidou, S., \& Zimányi, E. (2017). Time Series Databases and InfluxDB.

Nieles, M., Dempsey, K., \& Pillitteri, V. Y. (2017). An Introduction to Information Security. NIST Special Publication, 800, 12.

Perrone, G., \& Romano, S. P. (2017, September). The Docker Security Playground: A hands-on approach to the study of network security. In Principles, Systems and Applications of IP Telecommunications (IPTComm), 2017 (pp. 1-8). IEEE.

Sjöberg, J., Zhang, Q., Ljung, L., Benveniste, A., Delyon, B., Glorennec, P. Y. \& Juditsky, A. (1995). Nonlinear black-box modeling in system identification: a unified overview. Automatica, 31(12), 1691-1724.

Suricata, I. D. S. (2018). open-source IDS/IPS/NSM engine. Disponível em: http://suricata-ids.org/ - Ago 2018.

Taheri, J., Zomaya, A. Y., \& Kassler, A. (2017). vmBBProfiler: a black-box profiling approach to quantify sensitivity of virtual machines to shared cloud resources. Computing, 99(12), 1149-1177.

VirtualBox, O. (2018). General-purpose full virtualizer for x86 hardware. Disponível em: https://www.virtualbox.org. - Ago 2018.

Von Solms, R. (1998). Information security management (3): the code of practice for information security management (BS 7799). Information Management \& Computer Security, 6(5), 224-225. 